\title{
ON THE COMMENSURABILITY CLASS OF THE SIEGEL MODULAR GROUP
}

\author{
BY NELO D. ALLAN
}

\author{
Communicated by Murray Gerstenhaber, March 7, 1967
}

The purpose of this note is to determine the commensurability class of $G_{\mathrm{0}}$, where $G$ is either the Sympletic Group $\mathrm{Sp}_{n}$ or the Special Linear Group $\mathrm{Sl}_{n}$, and $\mathfrak{D}$ is the ring of integers of a number field $k$ of finite degree over the rationals. This problem has been solved in the local case by Hijikata [6], and Bruhat [3], for $\mathrm{Sl}_{n}$ and by Allan [1] and Bruhat-Tits [8] for $\mathrm{Sp}_{n}$. The only known global solution is due to Helling [5], in the case of $\mathrm{Sl}_{2}$. We shall exhibit a countable family of arithmetic groups in $G$, such that every maximal arithmetic group is conjugate to one group of the family, and if $o$ is a principal ideal ring, every group of this family is a maximal arithmetic group.

We know that if $G$ is a connected semisimple linear group defined over $k$, which is absolutely irreducible as a matrix group, then a maximal arithmetic group $\Delta$ is the normalizer of its intersection $\Delta^{\prime}$ with $G_{k}$, and $\Delta^{\prime}$ is selfnormalizer in $G_{k}$. Our problem is then to determine the selfnormalizer subgroups of $G_{k}$ whose normalizer is maximal, because the maximal groups which are the normalizer of maximal subgroups of $G_{k}$ has already been determined in [1], for the groups we are interested in.

Let $\mathfrak{p}$ be a finite prime spot of $k$ and $k(\mathfrak{p})$ be the completion of $k$ at $\mathfrak{p}$; let $\mathfrak{o}(\mathfrak{p})$ be the ring of integers of $k(\mathfrak{p})$. Let $\Delta$ be an arithmetic group contained in $G_{k}$ and let $\Delta^{\mathfrak{p}}$ be its p-adic closure in $G_{k(\mathfrak{p})}$. Assume that $N_{k}(\Delta)=\Delta$, and let $\Im$ be the ideal in $\boldsymbol{o}$ generated by all $\mu \in \mathcal{o}$ such that $\mu g$ has only algebraic integral entries for all $g \in N(\Delta)$. Let $\Omega(p)$ be the algebraic closure of $k(\mathfrak{p})$. It is easy to see that $N(\Delta)$ can be imbedded in $N\left(\Delta^{\mathfrak{p}}\right)$. Clearly the groups $\Delta$ which we want to determine are such that $\Delta=\cap\left(G_{k} \cap \Delta^{\mathfrak{p}}\right)$, the intersection taken over all finite primes of $k$. From now on we shall assume that all groups $\Delta$ considered, have this property. We shall also assume that $G$ has the strong approximation property (see [7]). Then it is very simple to verify the following two lemmas:

LEMMa 1. $N_{k}(\Delta)=\Delta$ if and only if $N_{k(\mathfrak{p})}\left(\Delta^{\mathfrak{p}}\right)=\Delta^{\mathfrak{p}}$ for all $\mathfrak{p}$. Similarly $\Delta$ is maximal in $G_{k}$ if and only if $\Delta^{\mathfrak{p}}$ is maximal in $G_{k(\mathfrak{p})}$ for all $\mathfrak{p}$.

Lemma $2 . \Delta$ is conjugate to $\Gamma$ in $G_{k}$ if and only if $(\Delta)^{\mathfrak{p}}$ is conjugate to $(\Gamma)^{\mathfrak{p}}$ in $G_{k(\mathfrak{p})}$ for all $\mathfrak{p}$. 
For each $\mathfrak{p}$, let $\Sigma_{\mathfrak{p}}$ be a finite set of open compact subgroups of $G_{k(\mathfrak{p})}$ such that

1. Every $\Delta$ in $\Sigma_{\mathfrak{p}}$ is selfnormalizer in $G_{k(\mathfrak{p})}$.

2. $N(\Delta)$ is maximal in $G_{\Omega(\mathfrak{p})}$, for all $\Delta \in \Sigma_{\mathfrak{p}}$.

3. If $\Delta^{\prime}$ is a maximal arithmetic group in $G_{\Omega(p)}$, then $\Delta^{\prime}=N(\Delta)$, where $\Delta$ is conjugate in $G_{k(p)}$ to some group in $\Sigma_{\mathfrak{p}}$.

Let $\Sigma$ be the set of all arithmetic groups in $G_{k}$, such that their $\mathfrak{p}$-adic completion lies in $\Sigma_{\mathfrak{p}}$ for all $\mathfrak{p}$.

THEOREM 1. If $\Delta^{\prime}$ is maximal arithmetic group in $G$, and $\Delta=\Delta^{\prime} \cap G_{k}$, then $\Delta^{\prime}=N(\Delta)$, and $\Delta$ is conjugate in $G_{k}$ to an arithmetic group in $\Sigma$.

The proof of this theorem is an immediate consequence of the above lemmas.

2. Case $G=\operatorname{Sl}_{n}(C)$. Let $\mathfrak{A}_{i j}, i, j=1, \cdots, n$ be $n^{2}$ fractional ideals in $k$ such that $\mathfrak{A}_{i j} \mathfrak{A}_{j k}=\mathfrak{A}_{i k}$, for all $i, j, k=1, \cdots, n$, and $\mathfrak{A}_{i j}$ is $n$th power free. Let $\mathfrak{B}_{i j}$ be integral ideals such that

1. $\mathfrak{B}_{i j}=\mathfrak{0}$ if $i \geqq j$ and $\mathfrak{B}_{i j} \mathfrak{B}_{j k}=\mathfrak{B}_{i k}$, for all $i, j, k=1, \cdots, n$.

2. $\mathfrak{B}_{i j} \mid \mathfrak{B}_{i m}$ and $\mathfrak{B}_{t j}$ for all $t \leqq i$ and $m \geqq j$, and $\mathfrak{B}_{i j}$ is square free for all $i, j=1, \cdots n$.

3. For each $\mathfrak{p} \mid \mathfrak{B}_{1 n}$ there exists $\delta \mid \mathfrak{B}_{1 n}$ such that if $s=s(\mathfrak{b})$ is defined by $\mathfrak{D} \mid \mathfrak{B}_{1 s+1}$ but $\mathfrak{D} \backslash \mathfrak{B}_{1 s}$, then $s \mid n, s(\mathfrak{p})=s(\mathfrak{D})$, and if $n=s(\mathfrak{D}) n(\mathfrak{D})$, then the ideal class of $b$ is an $n(\delta)$ th power of a some ideal class in $k$.

4. For all divisors $\mathfrak{D}$ of $\mathfrak{B}_{1 n}$ and all $m=1, \cdots, n(\mathfrak{D}), \mathfrak{b} \mid \mathfrak{B}_{i j}$ for all $i \leqq m s(d)$ and for all $j>m s(d)$.

Let $L(\mathfrak{P}, \mathfrak{B})$ be the direct summand order $L=\left(L_{i j}\right)$ where $L_{i j}$ $=\mathfrak{A}_{i j} \mathfrak{B}_{i j}$. We remark that $L(\mathfrak{D}, \mathfrak{B})^{\mathfrak{p}}$ the order obtained by Hijikata [5], and every order $L(\mathfrak{A}, \mathfrak{B})^{\mathfrak{p}}$ is conjugate to one of such orders in $\mathrm{Sl}_{n}(\Omega(\mathfrak{p}))$. Let $\Delta(\mathfrak{A}, \mathfrak{B})=L(\mathfrak{A}, \mathfrak{B}) \cap \mathrm{Sl}_{n}(k)$. Let $\Sigma^{\prime}$ the family of all $\Delta(\mathfrak{A}, \mathfrak{B})$, and let $\Sigma_{\mathfrak{p}}$ be the set of all $\Delta(\mathfrak{A}, \mathfrak{B})^{\mathfrak{p}}$. We observe that the class $\Sigma$ consists of all $\Delta(\mathfrak{A}, \mathfrak{B})$, where we drop the condition 3 for $\mathfrak{B}_{i j}$.

THEOREM 2. If $N_{k}(\Delta)=\Delta$ and $N(\Delta)$ is maximal in $G$, then $\Delta$ is conugate in $\mathrm{Sl}_{n}(k)$ to some $\Delta(\mathfrak{A}, \mathfrak{B}) \in \Sigma^{\prime}$.

2. $\Delta \in \Sigma^{\prime}$ then $N(\Delta)$ is maximal and $N(\Delta) / \Delta \simeq \mathcal{U}_{n} \times \mathfrak{C}_{n}\left(\mathfrak{B}_{1 n}\right) \times \mathcal{G}_{n}\left(\mathfrak{B}_{1 n}\right)$ (See [1], description of $N(\Delta) / \Delta$ ).

The proof is obtained from Theorem 1 and by showing that for each $\mathfrak{d} \mid \mathfrak{B}_{1 n}$ we can construct a matrix $g \in N(\Delta), g=\left(g_{i j}\right)$, such that $\left(g_{i j}\right)^{n(\mathfrak{b})}$ $=\left(\mathfrak{A}_{i j}^{\prime}\right)^{n(\delta)} / \delta$, where $C\left(\mathfrak{A}_{i j}\right)^{n(\downarrow)}=C(\delta)$. The proof of the maximality follows from the description of $N(\Delta) / \Delta$.

2. Case $G=\operatorname{Sp}_{n}(C)$. Let $\mathfrak{A}_{1}, \cdots, \mathfrak{A}_{n}$ and $\mathfrak{B}_{1}, \cdots, \mathfrak{B}_{n}$ be integral ideals such that

1. $\mathfrak{A}_{i} \mid \mathfrak{A}_{j}$ and $\mathfrak{B}_{i} \mid \mathfrak{B}_{j}$, for all $1 \leqq i \leqq j \leqq n$. 
2. $\mathfrak{A}_{n} / \mathfrak{P}_{1}$ and $\mathfrak{B}_{n} / \mathfrak{B}_{1}$ are square free and $\left(\mathfrak{B}_{n}, \mathfrak{X}_{n} / \mathfrak{A}_{1}\right)=1$. We shall assume that when $n=2 s$ is even, then $\mathfrak{B}_{s}=\mathfrak{B}_{s+1}$.

3. If $\mathfrak{q}$ is a nonprincipal prime, with $\mathfrak{q} \mid \mathfrak{B}_{n}$, then there exists $\mathfrak{d} \mid \mathfrak{B}_{n}$ such that $q \mid \delta$ and the ideal class of $\delta$ is a square.

We set

$$
\begin{aligned}
\mathfrak{L} & =\mathfrak{D} e_{1}+\cdots+\mathfrak{D} e_{n}+\mathfrak{A}_{1} \mathfrak{B}_{1} e_{n+1}+\cdots+\mathfrak{A}_{n} \mathfrak{B}_{n} e_{2 n} \text { and } \\
\mathfrak{L}^{\prime} & =\mathrm{D} e_{1}+\cdots+\mathfrak{D} e_{n}+\mathfrak{A}_{1} \mathfrak{B}_{1}^{\prime} e_{n+1}+\cdots+\mathfrak{A}_{n} \mathfrak{B}_{n}^{\prime} e_{2 n}
\end{aligned}
$$

where $\mathfrak{B}_{j}^{\prime}=\left(\mathfrak{B}_{n} / \mathfrak{B}_{n+1-j}\right)$. If $\mathfrak{D} \mid \mathfrak{B}_{n} / \mathfrak{B}_{1}$, we set $s=s(\mathfrak{D})$, the index $s$ such that $\delta \backslash \mathfrak{B}_{s} / \mathfrak{B}_{1}$ but $\mathfrak{D} \mid \mathfrak{B}_{s+1} / \mathfrak{B}_{1}$. If $n$ is odd, we set $\mathfrak{\Im}^{\prime}=\mathfrak{D}$ and if $n$ is even, say $n=2 s$, then we set $\Im^{\prime}$ be the product of all primes $\mathfrak{p}$ dividing $\mathfrak{A}_{s+1} / \mathfrak{A}_{s}$ such that there exists a divisor $\mathfrak{b}$ of this ideal such that $\mathfrak{p} \mid \mathfrak{b}$ and the ideal class of $\mathfrak{b}$ is a square. We shall denote by $\Delta(\mathfrak{A}, \mathfrak{B})$ the subgroup of $\operatorname{Sp}_{n}(k)$ consisting of all matrices $g \in \mathrm{Sp}_{n}(k)$ such that $g \mathfrak{L}=\mathscr{L}$ and $g \mathscr{L}^{\prime}=\mathscr{L}^{\prime}$.

THEOREM 3. If $\Delta$ is an arithmetic group in $\operatorname{Sp}_{n}(k)$ such that $N_{k}(\Delta)=\Delta$ and $N(\Delta)$ is maximal, then $\Delta$ is conjugate in $\mathrm{Sp}_{n}(k)$ to some $\Delta(\mathfrak{A}, \mathfrak{B})$.

2. If for all primes $\mathfrak{p}$ dividing $\mathfrak{B}_{n}$ such that it satisfies 3 , we have $s(\mathfrak{p})=s(\mathfrak{b})$, then $N(\Delta(\mathfrak{A}, \mathfrak{B}))$ is maximal, and $N(\Delta(\mathfrak{A}, \mathfrak{B})) / \Delta(\mathfrak{A}, \mathfrak{B})$ $\simeq \mathfrak{U}_{2} \times \mathfrak{C}_{2}(\Im) \times \mathcal{G}_{2}(\Im)$, where $\Im=\Im^{\prime} \mathfrak{B}_{n}$.

We shall sketch the proof. Let $\Sigma_{\mathfrak{p}}$ be the family consisting of all $\Delta(\mathfrak{A}, \mathfrak{B})^{\mathfrak{p}}$, hence $\Sigma$ is the family of all $\Delta(\mathfrak{A}, \mathfrak{B})$ obtained by dropping the condition 3 on $\mathfrak{B}_{n}$. We denote by $\Sigma^{\prime}$ be the family of all $\Delta(\mathfrak{A}, \mathfrak{B})$ and $\Sigma^{\prime \prime}$ be the subfamily consisting of those groups satisfying the hypothesis of the theorem. Our first assertion follows from Theorem 1 , and from the fact that if $\Delta(\mathfrak{A}, \mathfrak{B}) \in \Sigma$, then $N(\Delta(\mathfrak{A}, \mathfrak{B}))$ is maximal if and only if for all $\mathfrak{p} \mid \mathfrak{B}_{n}$ such that there is no $g \in N(\Delta(\mathfrak{A}, \mathfrak{B})), g=\left(g_{i j}\right)$ and 2 łord $_{\mathfrak{p}}\left(g_{i j}\right)^{2}$, then $\Delta(\mathfrak{A}, \mathfrak{B})^{\mathfrak{p}}$ is maximal in $\mathrm{Sp}_{n}(k(\mathfrak{p}))$. To get our second assertion for each $\delta \mid \Im$ such that the class of $b$ is a square, we construct an element $g$ in $N(\Delta(\mathfrak{A}, \mathfrak{B}))$ such that $g=\left(g_{i j}\right),\left(\left(g_{i j}\right)^{2}\right)$ $=\left(\mathfrak{A}_{i j}^{\prime}\right)^{2} / \mathfrak{D}$ for all $i, j=1, \cdots, 2 n$, where $\mathfrak{A}_{i j}$ are integral ideals.

We would like to point out that if $k$ has class number one, then $\Sigma=\Sigma^{\prime}=\Sigma^{\prime \prime}$. Also our family is not the smallest as possible, i.e., there are pairs of $\Delta \in \Sigma^{\prime}$ which are conjugate in $\operatorname{Sp}_{n}(C)$. Hence,

CoRollary. If $\Gamma$ is the Siegel Modular Group, then up to conjugacy in $\mathrm{Sp}_{n}(Q)$, the family of the normalizers in $\mathrm{Sp}_{n}(R)$ of the groups of $\Sigma$ consists of all maximal arithmetic groups in the commensurability class of $\Gamma$. If $\Delta(\mathfrak{A}, \mathfrak{B})$ lies in $\Sigma$, then the index of $\Delta(\mathfrak{A}, \mathfrak{B})$ in the normalizer of it in $\mathrm{Sp}_{n}(R)$ is $2^{a}$, where $a$ is the number of primes dividing $\Im$. 


\section{REFERENCES}

1. N. Allan, Arithmetic subgroups of some classical groups, Anais da Academia Brasileira de Ciencias (to appear).

2. - Maximal open-compact suhgroups of the projective symplectic group over a locally compact discrete valuation field, Pacific J. Math. (to appear).

3. F. Bruhat, "p-adic groups," in Algebraic groups and discontinuous subgroups, Proc. Sympos. Pure Math., Vol. 9, Amer. Math. Soc., Providence, R. I., 1966, pp. 63-70.

4. L. Gutnik, On the extension of integral subgroups of some groups, Vestnik Leningrad Univ. Ser. Math. Mech. and Astr. 19 (1957), 51-79.

5. H. Helling, Bestimmung der Kommensurabilitätsklasse der Hilbertschen Modulegruppe, Math. Z. 92 (1966), 269-280.

6. H. Hijikata, Maximal compact subgroups of p-adic classical groups, Sugaku no Ayumi 10-2, 1963, pp. 12-23. (Japanese)

7. M. Kneser, Einfach zusammenhängende algebraische Gruppen in der Arithmetik, Proc. Int. Congr. Math. Stockholm, (1962), pp. 260-263.

8. J. Tits and $\mathrm{F}$. Bruhat, BN-paires de type affine et données radicielles, $\mathrm{C} . \mathrm{R}$. Acad. Sci. Paris, 263 (1966), 766-769.

University of Notre DAME 